East African Medical Journal Vol. 77 No. 7 July 2000

PATTERN OF ANTICOAGULATION CONTROL AFTER HEART VALVE SURGERY AT THE KENYATTA NATIONAL HOSPITAL, NAIROBI S.W.O. Ogendo, MBChB, MMed, Senior Lecturer, Department of Surgery, College of Health Sciences, University of Nairobi, P.O. Box 19676, Nairobi.

\title{
PATTERN OF ANTICOAGULATION CONTROL AFTER HEART VALVE SURGERY AT THE KENYATTA NATIONAL HOSPITAL, NAIROBI
}

\author{
S.W.O. OGENDO
}

\begin{abstract}
Objective: To determine the pattern of anticoagulation control for post heart-valve surgery for patients on follow up at Kenyatta National Hospital(KNH).

Design: A combined prospective and restrospective hospital-based study. Retrospective period from January 1991 to 31st August 1997, while the prospective period was from 1st September 1997 to 31st November 1999.

Setting: Cardiothoracic surgery clinic, Kenyatta National Hospital, Nairobi.

Patients: Post heart valve surgery patients on warfarin and attending the cardiothoracic surgery clinic at Kenyatta National Hospital.

Main outcome measures: Clinic attendance intervals, average warfarin dosages, interval of dosage change, INR values and variations from accepted normal.

Results: A total of 103 patients fulfilled the criteria for inclusion into the study consisting of 77 mitral valve replacements, 18 aortic valve replacements, seven double valve replacements and one mitral valve repair. The total follow up time for the study period is $\mathbf{3 1 6 . 9}$ patients years. On average, patients attended their anticoagulation clinic once every 59 days. The average dose of warfarin prescribed was $6.81 \mathrm{mg}$ daily $( \pm 2.67 \mathrm{mg})$, with double valve replacement patients receiving a statistically significant lower dosage of $6.04 \mathrm{mg}( \pm 1.36 \mathrm{mg})$, (95\% confidence limits). On average, a warfarin dose change was made 1.48 times a year per patient. For all the patients, the mean INR was $2.50( \pm 1.18)$. The respective values for mitral, aortic, double valve replacement and the mitral repairs were $2.53( \pm 1.21), 2.32( \pm 1.04), 2.5$ $( \pm 1.05)$ and $2.02( \pm 0.53)$, respectively. Mitral valve repair patients maintained a significantly lower level of INR (95\% confidence limits). Only during $18 \%$ of the follow up time was adequate anticoagulation maintained. During the study period only $6.9 \%$ of patients were able to maintain adequate anticoagulation for $50 \%$ or more of their follow up time.

Conclusion: Anticoagulation control at the KNH still needs some improvements in clinic attendance and better dosage adjustments to achieve more appropriate INR values.
\end{abstract}

\section{INTRODUCTION}

Anticoagulation forms an essential part of the follow up of all patients with prosthetic heart valves with or without atrial fibrillation. The use of anticoagulation in this group of patients is now well established and essential to reduce morbidity associated with this state $(1,2)$. The relative risk varies with the different models of valves, (all of which have different haemodynamic characteristics), patients age, left atrial size, the presence of congestive cardiac failure and atrial fibrillation amongst other things(3).

In order to prevent thrombo-embolic phenomena anticoagulants are prescribed, of which warfarin is the most common. Appropriate levels of anticoagulation using warfarin are now universally monitored by the international normalised ration (INR).

The WHO introduced INR as a mode of standardising anticoagulation in the early 1980s(4) and this was subsequently adopted at the Kenyatta National Hospital (KNH), Nairobi in 1987. This standardisation became necessary due to the widely differing anticoagulation protocols and thromboplastins in place worldwide prior the time. The year 1991 was the first full year from when INR was used on a routine basis at the KNH.

The ideal level of anticoagulation in post-operative valve patients has been subject of a lot of debate over the years and was previously based on observational studies rather than scientific testing $(5,6)$. More recently the concept of valve specific anticoagulation is emerging as it has been realised that the thrombogenicity of different valves varies widely. The newer (or second generation) range of valves are generally less thrombogenic than their first generation counterparts.

Key to the efficient control anticoagulation is the formation of a reliable follow up service of the patients. Regular attendance by the patients, supplemented by a system of quality control anticaogulation are the prerequisites of such a service. In some centres where such quality control systems are in place, levels of adequate INR control maintained up to $80 \%$ of the patients attending the anticoagulation clinic for up to $60 \%$ or more their follow up time(8).

This study looks at the pattern of anticoagulation 
control at the KNH, Nairobi. The target population was the post heart valve surgery patients on anticoagulation attending the cardiothoracic surgery clinic.

\section{MATERIALS AND METHODS}

The study was carried out as a combined prospective and retrospective review of the pattern of clinic attendance and their anticoagulation control during the period from January 1991 to November 1999. Patient data were collected retrospectively from the patient files and anticoaguation control during the period from January 1991 to August 1997. Prospective data were obtained from follow up of the patients from September 1997 to November 1999.

Data collected from the patient files and anticoagulation booklets included details of the operative procedure, date of hospital discharge, all clinic attendance dates, INR estimation during each visit and warfarin dosage. Anticoagulation related morbidity and mortality data were not collected as this was a "preliminary data base" study. These details listed above were only documented for patients on follow up over the period from January 1991 onwards irrespective of the date of their surgery. All data collected (prospective and retrospectively) were amalgamated to make up a full data sheet for each patient.

All patient operated on at the $\mathrm{KNH}$ whose files could be traced in the record's office and those seen in the cardiothoracic surgery clinic were considered for inclusion into the study. The patients included in the study were those who had valve replacements and open valve repair procedures and on warfarin prophylaxis. Closed valvotomies and aortic ridge resections were not included.

Excluded from the study were patients attending the cardiothoracic clinic at $\mathrm{KNH}$ but had their first or subsequent open-heart surgery performed outside the KNH. Also excluded were those patients who either failed to attend postoperative clinic follow ups or made less than two postoperative clinic attendance post-discharge. The final group of patients excluded were those who though they attended follow up, ceased to do so prior to 1st January 1991, (the time from which INR estimation was utilised on a routine basis at the $\mathrm{KNH}$ ).

Data were analysed as mean, standard deviation, and confidence limits, the latter illustrated by box and whisker charts. Significance at a $95 \%$ level is taken as statistically significant.

The ranges of INR by the British Society of Haematologists for assessment of anticoagulant control were adopted for this study(9). These ranges are an INR level of less than two (or under anticoagulated), between 2 and 2.5, 2.5 to $3.0,3.0$ to 4.5 (the latter being the level previously recommended for all prosthetic valves) and finally greater than 4.5 (or over anticoagulated). Calculation of the durations in which the various of INR levels were maintained by the patients was done using the method of Duxbury, 1982(8). This method takes into account that not all INR estimations are done with the same interval in between tests.

\section{RESULTS}

Over the study period, a total of 103 patients fulfilled the criteria for inclusion into the study. There were 77 mitral valve patients, eighteen aortic patients, seven double valve patients and one mitral valve repair.

For all patients included, a total of 1836 clinic contacts were recorded making a total follow up period of 115,765 patient days(316.9 patient years). Out of these clinic visits, a total of $1,770(96.4 \%)$, of them had INR results available. A total of 13356 contacts (73.86\%), had warfarin doses retrieved.
For all patients combined the average interval between clinic visits at 58.95 days ( \pm 95.74 days). For the mitral valve replacement patients the average interval between clinic attendance is 57.06 days ( \pm 88.68 days) for aortic valve patients 58.22 days $( \pm 71.42)$ and the double valve replacement patients 76.24 days ( \pm 172.5 days). There was no statistically significant difference between the intervals of clinic visits for the four groups.

All patients reviewed were on warfarin medication. The mean dosage of warfarin prescribed for all the patients was $6.81 \mathrm{mg}$ daily $( \pm 2.67 \mathrm{mg})$. The respective figures of the patients with single mitral valve replacement, single aortic valve replacement and double valve were $6.77 \mathrm{mg}( \pm 2.8 \mathrm{mg})$, $7.32 \mathrm{mg}( \pm 2.17 \mathrm{mg})$ and $6.04 \mathrm{mg}( \pm 1.36 \mathrm{mg})$. The double valve replacement patients were receiving a statistically significant lower dose of warfarin than the other valve groups (Figure 1). The mitral valve repair patient was on warfarin but the dosage were inadvertently not recorded.

Figure 1

Mean warfarin dose prescribed per valve site

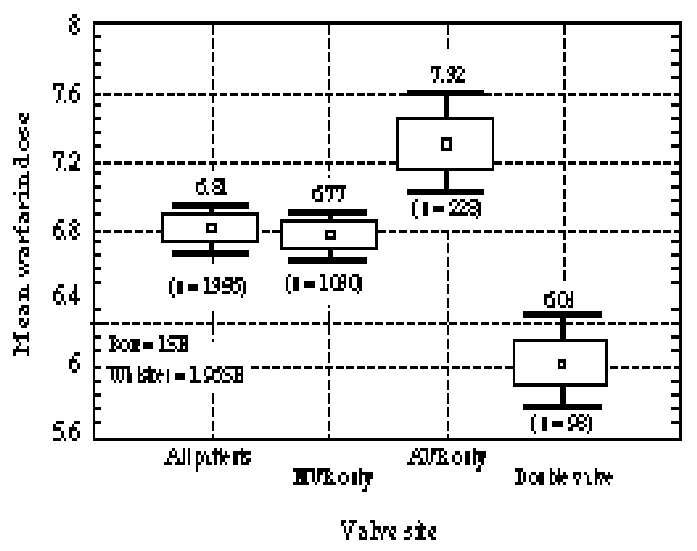

Figure 2

Mean INR for different valve sites

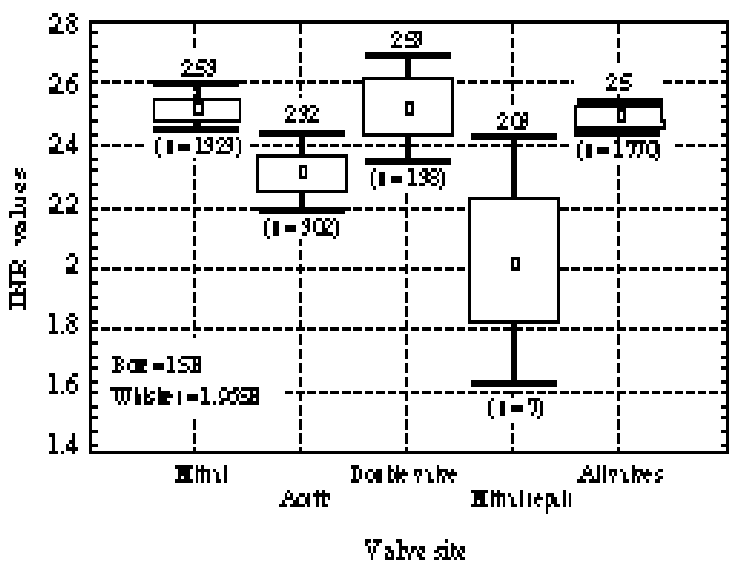


Figure 3

INR control in range 3.0-4.5 for valve patients

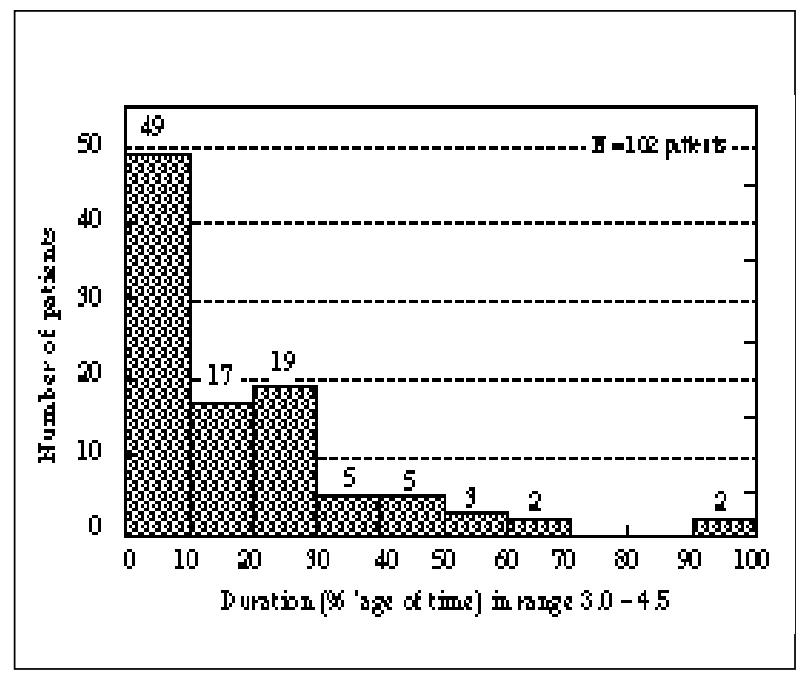

During the study period a total of 468 changes in the dosage of warfarin were made. The average number of warfarin dosage changes for all patients made per year was at 1.48 changes per patient per year .

For all INR values recorded the mean INR for all patients was $2.50( \pm 1.18)$ and the respective values for mitral, aortic, double valve patients and open mitral valve repair patients were $2.53( \pm 1.21), 2.32( \pm 1.04), 2.53$ $( \pm 1.05)$ and $2.03( \pm 0.53)$ respectively. The mitral valve repair patients had a significantly lower INR compared to the mitral valve replacement patients (Figure 2).

The duration in which various INR levels were controlled within the five ranges (less than 2, between 2 to $2.5>2.5$ and 3.0, > 3.0 and 4.5 and greater than 4.5), were $37.80 \%, 25.36 \%, 14.81 \%, 17.85 \%$ and $4.1 \%$ respectively (expressed as proportion to total study period). If we consider the ranges of INR 3.0 to 4.5 as the acceptable level of anticoagulation for mechanical valves, then for only $17.85 \%$ of the study period was an adequate level of anticoagulation maintained. The lone mitral valve repair patient is excluded from this analysis.

The prosthetic heart valves used at the $\mathrm{KNH}$ over the years have been of varying vintage. Most of the mechanical valves implanted(65.2\%) were of the first generation variety. However, the second - generation mechanical valves in this series were only used between May and June 1998 (Sorin bileaflet and Medtronic Hall valves). The total follow up for the second generation valves was only 9,526 patient days $(8.2 \%)$, out of the total follow up period. In view of the very low follow up rate of those valves, all the valves in this study have been considered to be of the first generation.

Forty nine patients $(48.0 \%)$ of the valve patients were able to maintain an adequate $\operatorname{INR}(3.0-4.5)$ for only $10 \%$ or less of the follow up time. While at the other extreme only seven patients or $6.9 \%$ of the patients were able to maintain an adequate INR control for $50 \%$ or more of the follow up time (Figure 3).

\section{DISCUSSION}

As a consequence of the high drop out rate to clinic follow up of post-heart valve surgery patients at the Kenyatta National Hospital(10), the immediate question that comes to mind is how effective is anticoagulation control in this group of patients who do not seem to be able to attend follow up reliably.

There has been no universally recommended period for the interval of follow up in-between clinic visits for patients on anticoagulation, and the cardiac unit of the KNH recommends six to eight weeks for patients whose INR is well controlled. For those out of control, more frequent appointments are given, especially for those over anticoagulated. It is important when deciding on a suitable duration between clinic visits to strike a balance between fine tuning of anticoagulation control and making economical usage of the anticoagulation clinic both in terms of cost and resources(8).

For this study the average duration between clinic visits of 59 days (six visits a year) fell outside our recommended range (with a wide standard deviation). In addition to the relatively high drop-out rate during follow up, the continuing patients only attended follow up six times a year on average.

In an effort to maintain fine-tuning of INR, two months may be too long an interval between clinic visits. This situation is however understandable in our set up as the economic realities make more frequent visits difficult for the vast majority of our patients. The difficulty here is both a problem of meeting the travelling expenses to Nairobi in addition to the clinic consultation and laboratory fees thereafter. Some patients do in fact request long clinic intervals for this very season. Similar problems have been noted in a similar population of patients from South Africa(11). The setting up of anticoagulation units outside Nairobi in the various provincial centres may help as a part solution to this aspect of the problem. Other factors often quoted for poor attendance in addition to finances include those patients not aware of the importance of regular follow up and those who, due to feeling better after surgery see no need to attend clinics on a regular basis(11).

There are probably a small number who do opt to seek private follow up outside the hospital. Unfortunately we have no records of this number but the author is sure that in view of the socio-economic group of our patients this number is no doubt very small. In fact a significant number of patients who have their surgery in the private setting often eventually opt for follow up at the KNH.

The majority of patients who attended follow up regularly filled in their follow up details on their follow up booklet diligently. This booklet is issued to all patients on warfarin upon discharge and may have resulted in the high $(96.4 \%$ and $73.9 \%)$ retrieval rates for the INR level and prescribed warfarin dosage respectively. The average dose of warfarin prescribed $(6.81 \mathrm{mg})$ was slightly less than that noted for a young age group by Routledge and 
co-workers(12). They also demonstrated that warfarin dosage fell with increase in age to about $50 \%$ in the elderly.

Nearly all the patients took less than $10 \mathrm{mg}$ daily to maintain their INR. However three patients within the study were on well over $10 \mathrm{mg}$ of warfarin with INR values persistently below 2.0. In addition to warfarin they were prescribed antiplatelet agents. Although antiplatelets may help in first generation valves, controversy still exists on the long-term use of antiplatelet agents with the modern valves $(11,12)$.

On a background of a significant level of under anticoagulation $(37.8 \%$ ), the average change in dosage of warfarin of three times in two years (or three times during twelve clinic visits), gives the impression of being an inadequate frequency and probably changes will need to be made more often. In view of a $73.9 \%$ data collection rate of warfarin dosages, there is possibility of underestimation of the true number of changes. The irregular patient attendances may also have significantly influenced this result. More regular and frequent clinic visits by the patients would probably give a more realistic picture.

There have been various recommendations of the optimal INR level for mechanical valves in the past. Initially the range $3.0-4.5$ for mechanical valves was proposed. Unfortunately these figures were based on population from the western world with differences to ours both in age and primary pathology. These figures were also derived from patients with a group of valve types of the first generation, which are associated with a thromboembolic rate twice that of second-generation prosthesis(14). It is generally accepted following recommendations of various individuals and organisations that ideal INR for first generation mechanical valves is of $3.0-4.5$ while for secondgeneration valves it is between 2.5 to $3.5(13,14)$. Butchart introduced the concept of prosthesis specific anticoagulation whereby the level of anticoagulation is tailored to the specific prosthesis and individual patient(3). Based on this concept, levels as low as 2.5 are used for Medtronic Hall valves in the isolated aortic position and 3.0 in the isolated mitral position(7). This gives a relatively good protection against thromboembolic episodes as well as avoiding the dangers of anticoagulant-induced bleeding. As a result of the wide variation of valves used in this hospital over the years, all valves in this study have been analysed as first generation valves. For this study in the absences of a local recommendation of ranges for INR, the recommendations of the British Society of Haematology have been adopted.

Based on these figures, for only $17.9 \%$ of the follow up time did our patients on average maintain adequate INR levels. While at the same time for $37.8 \%$ of the follow up time our patients were under anticoagulated, (INR less than 2). Only a very poor $6.9 \%$ of our patients were able to maintain an adequate level of INR control for $50 \%$ or more of the follow up time. This finding is not unexpected in view of poor long-term clinic follow up rates in this hospital combined with the relatively long inter-clinic visit interval.

In comparison, a similar figure demonstrating inadequate maintenance of anticoagulation during follow up of $12 \%$ was found in a comparable South African population of heart patients(11). This figure, like ours, is in sharp contrast to the best of centres with motivated patients who report adequate long-term anticoagulation rates up to $55-65 \%$ (15) of the follow up time.

"Adequate" anticoagulation by laboratory methods only may not necessarily translate into clinical outcome (thromboembolic phenomena, bleeding problems and mortality) which are actually the main objectives of any anticoagulation programme. A further study relating level of anitcoagulation and follow up for morbidity and mortality outcomes will be very useful in our set-up. There are a very small number of patients who self regulate their anticoagulation in our study group. One such patient in this group being a medical staff member in this very hospital. Patient regulated anticoagulation is used in some centres with varying results(11) though in this hospital with our patient population the author doubts whether we are ready for this, and would not recommend it with the exception of patients like the one mentioned with a medical background.

In addition to better patient follow up, therapeutic quality assessment is urgently needed in our clinic to improve the anticoagulation control. It has been found in many other centers, as in this study, that the main problem with anticoagulation is under anticoagulation. A clear lack of understanding of the management of INR by junior staff was identified as a significant factor contributing towards this. Unfortunately for a long time in our clinic the role of reviewing INR follow up has been left totally to them while the consultants reviewed the new cases. This observation has been noted in other hospitals as well, where junior staff tend to lean too much on the side of caution(11).

Apart from the patient-related factors affecting anticoagulation there are factors dependent on the hospital. These include the various hospital service charges, which can sometimes be a major problem in this part of the world. Concurrent prescription of other medications to the patient is a potential problem underscoring that prescribing habits of physicians is important(11). A change in the brand of warfarin issued by the pharmarcy is a common complaint by patients who note a sudden change in INR with the same dosage of warfarin. This is a newly emerging problem as the hospital moves more and more towards cheaper brands of drugs (and sometimes poorer quality), in order to meet its financial obligations.

In an effort to reduce errors arising as a result of physician factors as well as to reduce the mundane routine jobs in the clinic, computer aided anticoagulation has been used in some centres with good results indeed(16,11). 
This is a factor which we at a hospital like ours should not rule out as the capital outlay is well within the budgetary constraints of the hospital and would go a long way in relieving doctors from the routine job of regulating patient INR values. INR control can then be left to the nursing staff and the doctors only concentrate on those patients requiring special attention relating to their control. Such low labour intensive techniques with greater patient safely and accuracy have a lot of merit. Good results of INR control of $63 \%$ or more during follow up time have been achieved using this method(16).

In conclusion, the INR range of anticoagulation at the $\mathrm{KNH}$ mostly fall in the "under anticoagulated range". Further research relating this to morbidity and mortality in $\mathrm{KNH}$ is needed and appropriate measures taken. Areas to be considered are patient's attendance, staffing as well as the acquisition of equipment to reduce workload and at the same time increase the efficiency of services rendered. Improvement should be directed not only towards attaining better patient attendance of the clinic but also to educate patients on the importance of adequate control. A formal audit process to regularly evaluate progress and assess the subsequent improvement, if any, will also have to be put in place.

\section{ACKNOWLEDGEMENTS}

To the Director, Kenyatta National Hospital for allowing access to patient files and for permission to publish the study findings.

\section{REFERENCES}

1. Yeh, T.J., Anabtawi, I.N., Coenett, V.E. and Ellison, R.G., Influence of rhythm and anticoagulation upon the incidence of embolisation associated with Starr Edwards prothesis. Circulation 1967; 36(suppl. 1):77-81.

2. Effler, D.B., Favaloro. R. and Groves, L.K., Heart valve replacement: clinical experience. Ann. Thorac. Surg. 1965; 1:4- 24.

3. Butchart, E.G., Lewis, P.A., Bethal, J.A. and Breckenridge I.M. Adjusting anticoagulation to prosthesis thrombogenisity and patient risk factors: recommendation for the Medtronic Hall valve. Circulation 1991; 84(suppl. iv): 61-69.

4. WHO expert committee on Biological Standardisation. Standardisation in the control of anticoagulation (oral). 28th report. Tech Rep. Ser. 610, Geneva, World Health Organisation, 1977; 45-51.

5. Hirsh, J., Poller, L., Deykin, D., Levine, M. and Dalen, J.E. Optimal therapeutic range of oral anticoagulation. Chest 1989; 95: 5S-11S.

6. Sacket, D.L. Rules of evidence and clinical recommendations on the use of antithrombotic agents. Chest 1989; 95:2S-4S.

7. Butchart, E.G., Lewis, P.A., Grunkemeir, G.T. et al. Low risk thrombosis and serious embolic events despite low intensity anticoagulation. Experience with 1,004 Medtronic Hall valves. Circulation 1988; 78(suppl. 1): 66-77.

8. McD, Duxbury, B. Therapeutic control of anticoagulation treatment. Brit. Med. J. 1982; 284:702-704.

9. British Society of Haematology, British Committee for standards in Haematoloty, Haemostasis and Thrombosis Task Force. Guidelines and oral anticoagulation, 2nd edition. J. Clin. Pathol. 1990; 43:177-183.

10. Ogendo, S.W.O. Follow up of patients after open heart surgery. East Afr. Med. J. 1998; 75:675-678.

11. Eric, G., Butchart, Endre Bodnar. Thrombosis, Embolism and bleeding. ICR Publications London. First edition 1992; 362-384.

12. Routledge, P.A., Chapman, P.H., Davies, D.M. and Rawlins, M.D. Factors affecting warfaring requirements. Europ. J. Clin. Pharmacol. 1979; 15:319-322.

13. Armand, P. and Westerby, S. Surgery of acquired aortic valve disease. ISIS Medical Media Ltd. First edition 1997; 296-303.

14. Hirsh, J., Dalen, J.E., Deykin, D. and Poller, L. Oral anticoagulants; mechanisms of action, clinical effectiveness, and optimal therapeutic range. Chest 1992; 102:312S-326S.

15. Hirsh, J., Fuster, V. Guide to anticoagulant therapy. Part 2: Oral anticoagulants. AHA Medial/Scientific Statement special report. Circulation 1994; 89:1469-1480.

16. Brotman, L. Anticoagulation in myocardial infarction. Amer. J. Cardiol. 1958; 81:260 -270.

17. Poller, L., Shiach, C.R., MacCallum, P.K., Johansen, A.M., Münister, A.M., Magaihaes, A. and Jespersen, J. On behalf of the European Concerted Action on Abticoagulation. Multicentere randomised study of computerised anticoagulant dosage. Lancet 1998; 353:1505-1509. 